\title{
Topological Invariants from Higher Categories
}
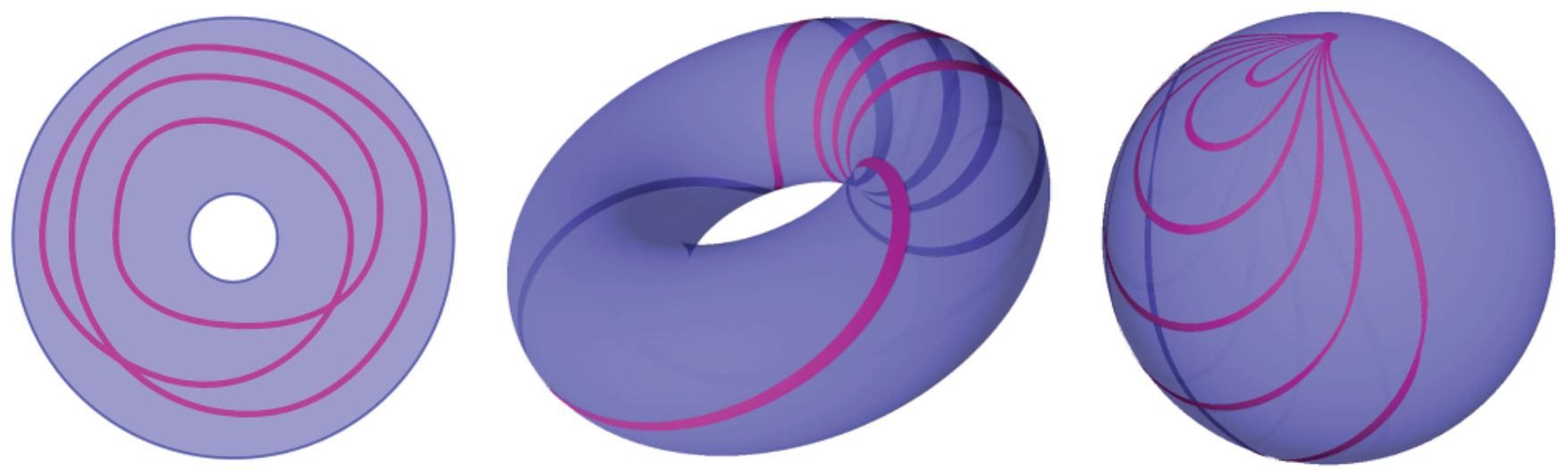

\section{Nick Gurski, Niles Johnson, and Angélica M. Osorno}

While invariants of geometric or topological objects can be of any sort, most modern invariants are algebraic in nature. The goal of this article is to survey some of the ways in which categorical algebra can be utilized to measure topological phenomena. This area of mathematics is a relatively recent outcrop on a vast landscape of interactions between algebra and geometry. The critical points of a differentiable function or the Betti numbers and Euler characteristic of a topological space are two among many points of interest on this landscape, and the field of algebraic topology has its origins nearby. Venturing further, Betti numbers were recognized by Noether and Vietoris as the avatars of homology groups, a perspective that was not immediately adopted but whose advantages are now clear. Our route begins here and leads toward higher-categorical algebra and the topological applications thereof.

Just as the theory of groups provides an abstraction and formalism for mathematical structures observed in many examples (e.g., symmetry or additivity), so the theory of

Nick Gurski is an assistant professor at Case Western Reserve University. His email address is nick.gurski@case.edu.

Niles Johnson is an associate professor at Ohio State University, Newark. His email address is niles@math. osu. edu.

Angélica Osorno is an associate professor at Reed College. Her email address is aosorno@reed.edu.

Communicated by Notices Associate Editor Stephan Ramon Garcia.

For permission to reprint this article, please contact:

reprint-permission@ams.org.

DOI: https://doi.org/10.1090/noti1934 categories abstracts and formalizes mathematical structure observed in many applications (e.g., functoriality or naturality). More recently, categories with additional structure (monoidal, tensor, fusion, enriched, etc.) have found applications in geometry, representation theory, and physics. Some of these applications have their roots in the deep connections between topological invariants and categorical structure. Our discussion below will give an overview of these connections, outlining several of the key ideas but focusing on stability (in topology) and symmetric monoidal structure (in category theory).

A natural history of category theory. Even before category theory emerged as a distinct mathematical perspective, the importance of functoriality-that some constructions are preserved by morphisms - had been observed in various settings. For example, given a ring $R$ we can construct the ring of polynomials $R[x]$ with coefficients in $R$, and any ring homomorphism $f: R \rightarrow S$ will induce a ring homomorphism $f_{*}: R[x] \rightarrow S[x]$ by applying $f$ to the coefficients. Similarly, the construction of the dual $V^{*}$ of a vector space $V$ applies to morphisms as well as to objects, in the sense that any linear transformation $T: V \rightarrow$ $W$ induces a linear transformation (in the opposite direction!) $T^{*}: W^{*} \rightarrow V^{*}$.

Functoriality of the fundamental group is what proves that there is no retraction of a disk $D^{2}$ to its boundary circle $S^{1}$. Such a retraction 
would necessarily induce a retraction on fundamental groups

$$
\mathbb{Z} \rightarrow 0 \rightarrow \mathbb{Z}
$$

such that the composite is equal to the identity homomorphism on the integers. This produces a contradiction, so no such retraction can exist. This argument shows the utility of a construction that preserves composition and identities - the very definition of functoriality.

Eilenberg and Mac Lane introduced category theory per se to formalize the notion of natural transformation between functors. Recall that a category $\mathscr{C}$ has objects and morphisms, which we draw as vertices and directed edges, together with a composition law for morphisms and identity morphisms satisfying associativity and unit axioms. Functors are maps between categories, consisting of a function sending objects to objects and a function sending morphisms to morphisms, preserving composition and identities. Natural transformations are then morphisms between functors. One early motivation was to codify the properties of the suspension isomorphism for generalized (co)homology theories (see (2)), but natural transformations appear in every domain that functors do. For example, there is always a ring homomorphism including $R$ into $R[X]$ as the subring of constant polynomials. This homomorphism is constructed "naturally" in the sense that it commutes with all ring homomorphisms: the two composites around the left square in Figure 1 are equal.

Likewise, there is an injective linear transformation from a vector space to its double dual, which is an isomorphism if the vector space is finite-dimensional. This transformation is "naturally" constructed in a way that is uniform for any vector space and independent of any choice of basis. Concretely, this means that the two composites around the right square in Figure 1 are always equal for any linear transformation $T$.

Category theory provides a language, a context, and indeed a mathematical theory that abstracts the general properties of such constructions from their specific instances. Thus, category theory is sometimes called "the mathematics of mathematics" for its role in organizing general features across mathematical disciplines.

Another point of view on category theory emphasizes its algebraic nature, by which we mean that it involves data (objects, morphisms, functors, transformations), rules for combining that data (identities, composition, etc.), and axioms such as the associativity of composition or the equalities in naturality squares. For example, a group $G$ can be considered as a category consisting of a single object whose set of endomorphisms is given precisely by $G$. Thus, we can think of categories as generalizations of monoids or groups and hence as algebraic objects in their own right.

Such a generalization is the start of what is sometimes called higher-dimensional algebra. Thinking of a set as a col-
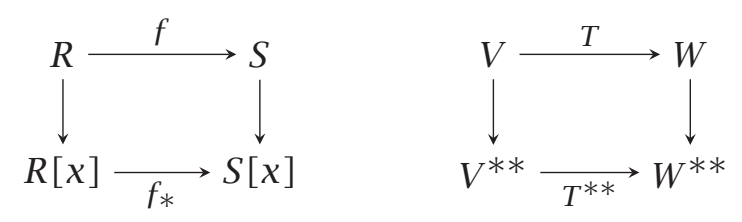

Figure 1. Naturality squares.

lection of 0-dimensional elements, the multiplication of group elements or the addition of vectors is thought of as 0dimensional algebra. A category has both 0-dimensional and 1-dimensional aspects-the objects and morphismsso any algebraic structure on a category is inherently 1dimensional. For example, the direct sum of vector spaces is additive on dimension and thus provides a higherdimensional analogue of the sum of natural numbers.

The pattern of organizational framework repurposed as independent theory leading to higher-dimensional invariants repeats within category theory itself. Much of the power of basic category theory arises from the use of natural transformations. In turn, many of those arguments can be abstracted and formalized in the context of 2-categories, in which we have 2-dimensional arrows between morphisms.

This process can be continued indefinitely and indeed has spawned the entire subject of higher-dimensional category theory: the study of structures that have objects (thought of as 0-dimensional points), morphisms between them (thought of as 1-dimensional arrows), 2-dimensional morphisms between those, and so on, together with composition and identity operations. Like 1-dimensional category theory, the motivations for developing this higherdimensional version come from topology, algebraic geometry, logic, physics, computer science, and the subject itself.

An analogy with the theory of groups. Our two perspectives on category theory-as an organizing abstraction and as a higher-dimensional algebra-are themselves generalizations of two perspectives on group theory. From one point of view, groups are an abstraction of symmetries. Homomorphisms between symmetry groups give comparisons between symmetries of one object and those of another. Permutations of polynomial roots (Galois theory) and symmetries of manifolds (Klein's Erlangen program) are two early and formative examples of this perspective. But we also understand groups as useful algebraic invariants in their own right. Mathematical invariants that are groups instead of mere numbers will detect more subtle phenomena.

Example 1. For each natural number $n$ we have a symmetric group $S_{n}$. This can be thought of as a 1-object category, where the object is an $n$-element set and $S_{n}$ is the set of automorphisms of it. When we put all of these objects together in a single category, $\mathbf{S}$, we have an object for 


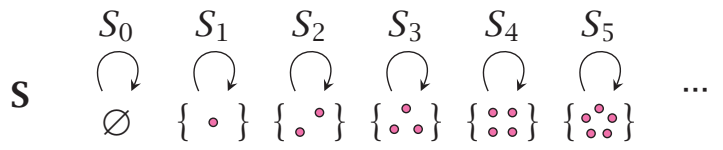

Figure 2. The category $\mathrm{S}$ has objects given by the natural numbers and automorphisms given by symmetric groups.

each $n \geq 0$ and each object has automorphisms given by its symmetric group. See Figure 2.

This category has further structure given by the disjoint union of sets and the block sum of permutations. This endows $\mathbf{S}$ with an additive structure that, on objects, is given by the addition of natural numbers. In this way $\mathbf{S}$ is a higher-dimensional analogue of the natural numbers, encoding both cardinality and permutations of elements.

The category $\mathbf{S}$ is an example of a symmetric monoidal category using the addition defined above, a structure we will focus on in the latter sections. Symmetric monoidal categories abound; the category of sets, Sets, with cartesian product is one, and the category of vector spaces, Vect, with tensor product is another.

The purpose of this note is to explain how symmetric monoidal structure in higher category theory corresponds to stability in topology. We begin with a description of some classical invariants in algebraic topology and explain how these are encoded in the categorical algebra of weak $n$-groupoids. Then we explain stabilization in topology and how the corresponding categorical objects arise from symmetrization. We describe several ways that interesting topological invariants motivate natural and equally interesting categorical incarnations, focusing on the data arising from Postnikov towers in topology. The mathematics we explain here falls under the broad umbrella of the stable homotopy hypothesis, which we outline over the course of the article and detail in the section "LowDimensional Examples of the Stable Homotopy Hypothesis."

\section{The Fundamental Group(oid) and Classifying Space}

The fundamental group(oid). The fundamental group of a topological space was introduced in 1895 by Poincaré in Analysis Situs. For a space $X$ and a basepoint $x \in X$, this is the group $\pi_{1}(X, X)$ formed by homotopy classes of paths in $X$ beginning and ending at $X$. The fundamental group measures holes of a certain type in a space, as Example 2 demonstrates.

Example 2. The fundamental group of the circle $S^{1}$ is isomorphic to the group of integers, as is that of an annulus $S^{1} \times[0,1]$. A loop is characterized by its (signed) winding number around the circle. The fundamental group of the torus $S^{1} \times S^{1}$ is $\mathbb{Z} \times \mathbb{Z}$, and a loop is characterized by its winding numbers around the two coordinate circles. The
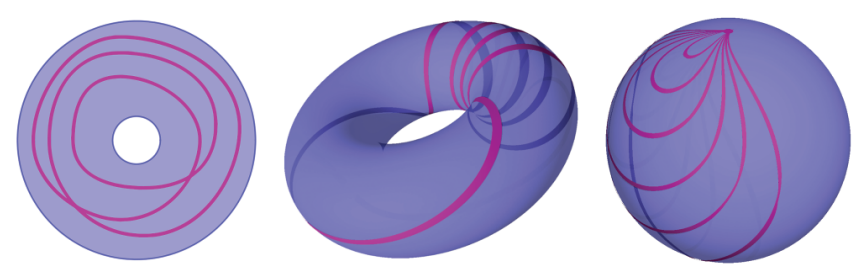

Figure 3. Left: a loop with winding number 3 in the annulus; Middle: a loop with winding number $(1,5)$ in the torus; Right: contractible loops in the 2-sphere.

fundamental group of the 2-sphere $S^{2}$ is trivial because any loop there can be contracted to a point. See Figure 3 for these examples.

Note that the definition of $\pi_{1}$ depends on a choice of basepoint for the loops. A path between two points gives rise to an isomorphism between the fundamental groups based at those points, but different paths can produce different isomorphisms and different path components can have different fundamental groups. Thus, the fundamental group fails to be a functor on the category of topological spaces. One can either work with based spaces or track the dependence on basepoints with a higher-categorical structure. One such structure is the fundamental groupoid $\Pi_{1} X$ of a space $X$.

The objects of the fundamental groupoid $\Pi_{1} X$ are given by the points of $X$, and the morphisms between two points $x$ and $y$ are the homotopy classes of paths between them. The term groupoid is used for a category in which every morphism is invertible, and $\Pi_{1} X$ is a groupoid because paths are reversible. The fundamental group based at a point $X$ is recovered as the group of endomorphisms of $x$ (all of which are automorphisms) in the fundamental groupoid. Moreover, the change-of-basepoint isomorphisms are encoded as morphisms in $\Pi_{1} X$, and the set of isomorphism classes of objects is the set $\pi_{0}(X)$ of path-connected components of $X$. At first sight, this may seem to be merely a clever reframing of elementary notions, but the key feature is that it allows these two invariants-the set of components and the fundamental group(s) thereof-to be encoded as a single algebraic object. Unfortunately this is all the information $\Pi_{1} X$ carries, so we will need more sophisticated invariants to carry additional topological information.

The classifying space. Whereas the fundamental groupoid makes a category out of a space, taking paths for morphisms, the classifying space constructs a space from a category. Given a category $C$, the classifying space $B C$ (Figure 4 ) is constructed as a CW complex with 0 -cells given by the objects of $C, 1$-cells given by morphisms, 2-cells given by composable pairs of morphisms, and, in general, $n$-cells given by composable $n$-tuples of morphisms. In particular, every morphism in $C$ gives a path in $B C$. This 


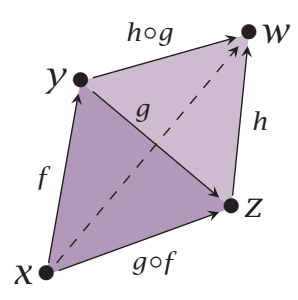

Figure 4. The classifying space of a category is constructed as a CW complex with cells given by composable morphisms.

construction generalizes the notion of classifying space for a group, where a group is regarded as a 1-object category.

The set $\pi_{0}(B C)$ of connected components of $B C$ is isomorphic to the set of connected components of the underlying graph of objects and arrows in $C$. For an object $X$ in $C$, the fundamental group $\pi_{1}(B C, x)$ has elements that are represented by equivalence classes of zigzags of morphisms that start and end at $X$,

$$
x \stackrel{f_{1}}{\rightarrow} x_{1} \stackrel{f_{2}}{\leftarrow} x_{2} \stackrel{f_{3}}{\rightarrow} \cdots \stackrel{f_{n}}{\leftarrow} x_{n} \stackrel{f_{n+1}}{\rightarrow} x,
$$

with relations induced by composition. A crucial distinction between $C$ and $B C$ is that the latter loses the notion of directionality of morphisms. If $C$ is a groupoid, then zigzags of morphisms can be replaced by composites in $C$ using the inverses of the "wrong way" morphisms. Such a replacement procedure proves that, for a groupoid, the fundamental group $\pi_{1}(B C, x)$ is isomorphic to $C(x, x)$, the group of automorphisms of $x$.

This connection can be expressed more clearly in categorical language. For a general category $C$, there is a natural transformation $C \rightarrow \Pi_{1} B C$ known as groupoid completion, the universal way to invert all morphisms in $C$. If $C$ is a groupoid, then $C \rightarrow \Pi_{1} B C$ is an equivalence of categories.

The reader might wonder now about the relationship between $X$ and $B \Pi_{1} X$ since they share $\pi_{0}$ and $\pi_{1}$. Spaces have more homotopy groups than these two, and moreover they are assembled in complicated ways. This additional topological information is all controlled by the Postnikov tower, which we explain in the next section. To capture more data in the tower using categorical tools, we explore in the section "Higher Groupoids, More Subtle Algebra" a higher-dimensional analogue of $\Pi_{1}$ that faithfully reflects more of the Postnikov tower.

\section{Higher Homotopy Groups, More Subtle Invariants}

Loop spaces and higher homotopy groups. Further topological information beyond the set of connected components and their fundamental groups is encoded in the higher homotopy groups of a space.

Definition 3. For based spaces $X, Y$, we let $[X, Y]$ denote the set of based homotopy classes of continuous based maps $X \rightarrow Y$.

The group $\pi_{n}(X)$ is given by the set of based homotopy classes of maps from the $n$-sphere $S^{n}$ into $X$, written $\left[S^{n}, X\right]$. As with $\pi_{1}$, this involves a choice of basepoint that is often suppressed in the notation; paths between different basepoints induce isomorphisms on $\pi_{n}$ as in the case $n=1$. We restrict to path-connected spaces for the remainder of this section.

For a based space $X$, we denote by $\Omega^{n} X$ the space of based maps from $S^{n}$ to $X$. When $n=1$ this is called the loop space of $X$ and is simply denoted $\Omega X$. The elements of the group $\pi_{n}(X)$ are the path components of $\Omega^{n} X$, and the group structure on $\pi_{n}(X)$ (abelian if $n>1$ ) is induced by an up-to-homotopy group structure on $\Omega^{n} X$. One can show, using the loop-suspension adjunction (1) below, that the loop space shifts homotopy groups:

$$
\pi_{n}(\Omega X) \cong \pi_{n+1}(X) \text {. }
$$

These elementary observations are the first signs of a deeper theory that we will introduce in the sections "Stabilization in Topology" and "The Algebra of Iterated Loop Spaces."

A map $f$ that is a homotopy equivalence induces isomorphisms on homotopy groups. ${ }^{1}$ A homotopy type is an equivalence class of spaces under the relation of homotopy equivalence; i.e., $X$ and $Y$ have the same homotopy type if there is a homotopy equivalence between them. Although the homotopy type of a space $X$ determines its homotopy groups, the converse is generally not true; an abstract isomorphism of homotopy groups does not necessarily induce a homotopy equivalence. One also needs attaching information, akin to extension classes in group theory. As we now explain, one can approach the classification of homotopy types from the point of view of basic blocks, the Eilenberg-Mac Lane spaces, and attaching data known as Postnikov invariants.

Definition 4. For $n \geq 0$ and a group $A$ (abelian if $n \geq$ $2)$, the $n$th Eilenberg-MacLane space $K(A, n)$ is a space whose homotopy groups are zero except in dimension $n$, and $\pi_{n}(K(A, n)) \cong A$.

There are many ways to construct such a space, but all of them result in homotopy equivalent spaces. Note that because the loop space construction shifts homotopy groups, $\Omega K(A, n+1)$ is an Eilenberg-Mac Lane space for $A$ in dimension $n$. Uniqueness therefore implies that $K(A, n) \simeq$ $\Omega K(A, n+1)$.

Given a space $Z$, we say that a space $W$ is a delooping of $Z$ if there is a homotopy equivalence $Z \simeq \Omega W$. Note that both the space $W$ and the homotopy equivalence may not be unique. The previous paragraph explains that $K(A, n+$ $1)$ is a delooping of $K(A, n)$.

\footnotetext{
${ }^{1}$ The converse is not true in general but does hold for $\mathrm{CW}$ complexes.
} 
Eilenberg-Mac Lane spaces are closely connected with ordinary cohomology, as described in the following theorem. ${ }^{2}$

Theorem 5. Cohomology is represented by homotopy classes of maps into Eilenberg-Mac Lane spaces. More precisely, for each natural number $n>0$, based space $X$, and abelian group $A$ there are isomorphisms

$$
H^{n}(X ; A) \cong[X, K(A, n)]
$$

natural in $X$ and $A$.

One can use Eilenberg-Mac Lane spaces to construct a space with any given sequence of homotopy groups $A_{i}$ provided $A_{i}$ is abelian for $i \geq 2$. Indeed, the product

$$
P=\prod_{i} K\left(A_{i}, i\right)
$$

has $\pi_{i}(P) \cong A_{i}$. Given a space $X$, one can ask whether it is different from a product of Eilenberg-Mac Lane spaces. For example, if $X$ has just two nontrivial homotopy groups, $\pi_{1}=A_{1}$ and $\pi_{2}=A_{2}$, then $X$ sits in a fiber sequence

$$
K\left(A_{2}, 2\right) \rightarrow X \rightarrow K\left(A_{1}, 1\right)
$$

where the first of these maps is an isomorphism on $\pi_{2}$, and the second is an isomorphism on $\pi_{1}$.

There is an action of $\pi_{1}(X)=A_{1}$ on $\pi_{2}(X)=A_{2}$ induced by the fibration structure, and the homotopy type of $X$ depends subtly on this action. However, in the context we study below, this action is necessarily trivial, and therefore for the remainder of this section we assume that $\pi_{1}(X)$ acts trivially on all homotopy groups of $X$. Such spaces are called simple.

The general theory of fibrations then gives a continuation of this sequence to the right:

$$
K\left(A_{2}, 2\right) \rightarrow X \rightarrow K\left(A_{1}, 1\right) \stackrel{k_{1}}{\rightarrow} K\left(A_{2}, 3\right) .
$$

The map denoted $k_{1}$ corresponds to a cohomology class in

$$
H^{3}\left(A_{1} ; A_{2}\right) \cong\left[K\left(A_{1}, 1\right), K\left(A_{2}, 3\right)\right],
$$

which we also denote $k_{1}$, and it classifies spaces with two nontrivial homotopy groups in the following sense.

Theorem 6. The total space $X$ above decomposes as a product $K\left(A_{1}, 1\right) \times K\left(A_{2}, 2\right)$ if and only if $\pi_{1}(X)$ acts trivially on $\pi_{2}(X)$ and $k_{1}=0$ in $H^{3}\left(A_{1} ; A_{2}\right)$. More generally, the homotopy type of such a space is determined by the two groups and the cohomology class $k_{1}$.

\footnotetext{
${ }^{2}$ We state this theorem only for $n>0$ so as to avoid the distinction between reduced and unreduced cohomology, which agree in positive degrees. It is the reduced cohomology groups that are represented by Eilenberg-Mac Lane spaces, and we implicitly take reduced cohomology throughout this article.
}

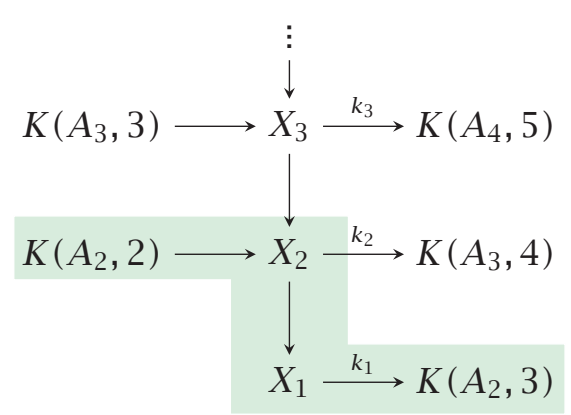

Figure 5. Postnikov tower for a connected space $X$. The highlighted region connecting two different layers is a homotopy fibration sequence.

Postnikov towers. This map $k_{1}$ is called the Postnikov invariant at level 1 , and our discussion above can be extended for higher homotopy groups as in Figure 5. For each $n$, we have a map $X \rightarrow X_{n}$ inducing an isomorphism on homotopy groups $\pi_{k}$ for $k \leq n$. The space $X_{n}$ is called the $n$th Postnikov truncation or the homotopy $n$-type of $X$. The homotopy groups $\pi_{k}\left(X_{n}\right)$ are zero for $k>n$, so $X_{1} \simeq K\left(A_{1}, 1\right)$. The sequence of spaces $X_{n}$ and cohomology classes $k_{n}$ determines this entire diagram, called the Postnikov tower of $X$. The central result of Postnikov theory can be summed up by the following slogan.

Slogan 7. The homotopy type of a space $X$ is determined by its homotopy groups and Postnikov invariants.

The Postnikov tower presents a connected topological space in terms of its homotopy groups and the cohomology classes along which they are attached. This is dual to the cellular construction of a space, which proceeds by attaching disks and thus presents a space in terms of its homology groups and the homotopy classes along which elements are attached.

\section{Higher Groupoids, More Subtle Algebra}

For a space $X$, the fundamental groupoid $\Pi_{1}(X)$ provides an algebraic model for the homotopy 1-type of $X$. More precisely, the first Postnikov truncation $X_{1}$ and $B \Pi_{1} X$ are homotopy equivalent. Moving up the Postnikov tower, we seek algebraic models for the higher homotopy groups and Postnikov invariants.

There is a striking result by Thomason that every homotopy type can be constructed as the classifying space $B C$ for some category $C$. However, the higher Postnikov data of $B C$ is difficult to extract from $C$ itself. If $C$ is a groupoid this is easily done, because $\pi_{n}(B C, x)=0$ for all $n \geq 2$ and all basepoints $x$. In this section we discuss notions of higher groupoid which provide algebraic models for higher-dimensional Postnikov data, but still in a finite range. 


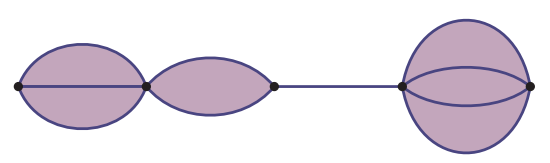

Figure 6. An example of composition formed by attaching disks and intervals, respectively, along intervals and points.

The fundamental $n$-groupoid $\Pi_{n}$. Recall that the groupoid $\Pi_{1} X$ has morphisms given by the homotopy classes of paths in $X$, and these are classes of continuous maps from the 1-dimensional disk $D^{1}$ to $X$. Evaluation at the endpoints gives the source and target of a morphism. Thus, we seek a higher-dimensional analogue of the fundamental groupoid that includes the data of maps $D^{i} \rightarrow X$ for higher-dimensional disks $D^{i}$.

Desideratum 8. The fundamental $n$-groupoid $\Pi_{n} X$ of a space $X$ has as data

- all continuous maps $D^{i} \rightarrow X$ for $0 \leq i<n$,

- all homotopy classes of maps $D^{n} \rightarrow X$ (relative to the boundary), and

- information for how these maps or classes of maps glue together along compatible boundaries.

These compatible boundaries are given by the two inclusions $\sigma, \tau: D^{i-1} \rightarrow D^{i}$ as the northern and southern hemispheres of the boundary of $D^{i}$ and their iterates. We can extract from this some basic features of an algebraic structure we would like to call an $n$-groupoid. Letting $G_{i}$ denote the set of all continuous maps $D^{i} \rightarrow X$ for $i<n$ and the set of homotopy classes of maps $D^{n} \rightarrow X$ relative to the boundary when $i=n$, there are source and target maps $s, t: G_{i} \rightarrow G_{k}$ induced by the iterations of $\sigma$ and $\tau$. Abstracting this structure leads to the following.

\section{Desideratum 9. An $n$-groupoid $G$ has}

- sets $G_{i}$ of $i$-cells for $i=0, \ldots, n$;

- maps $s, t: G_{i} \rightarrow G_{i-1}$ assigning to each $i$-cell both a source and a target $(i-1)$-dimensional cell;

- maps $G_{i} \rightarrow G_{i+1}$ that assign to each $i$-cell $\alpha$ an identity $(i+1)$-cell with $\alpha$ as both source and target; and

- a variety of composition laws ${ } k$, subject to further associativity, invertibility, and unit axioms.

The composition laws are the most complicated feature of such a definition, but also the most interesting. They are of the form $\circ_{k}: G_{i} \times_{k} G_{i} \rightarrow G_{i}$, where $G_{i} \times_{k} G_{i}$ is the subset of $G_{i} \times G_{i}$ in which the $k$-dimensional target of the first cell matches the $k$-dimensional source of the second cell. Topologically, these functions show the different ways to attach two disks together along some lower-dimensional boundary disk; see for example Figure 6 .
The homotopy hypothesis. What we have described so far is not a rigorously defined structure, only some basic desiderata. The simplest definition fulfilling these yields the notion of strict $n$-groupoid, in which the associativity and unit axioms for composition hold in each dimension separately. The definition of strict $n$-groupoid is most succinctly stated using the theory of enriched categories: a strict $n$-groupoid is a groupoid enriched in strict $(n-1)$ groupoids. We will not give further details here, as this definition has a serious defect, first described by Carlos Simpson.

Theorem 10. Topological spaces that correspond to strict 3groupoids have trivial Whitehead products. In particular, strict 3-groupoids fail to model all homotopy 3-types.

In order to understand the statement of this theorem, note that the homotopy groups of a space are not just a sequence of independently defined groups but have operations between them. One such operation arises from a canonical map

$$
S^{k+j-1} \rightarrow S^{k} \vee S^{j}
$$

This yields a bilinear function $\pi_{k}(X) \times \pi_{j}(X) \rightarrow$ $\pi_{k+j-1}(X)$ known as the Whitehead product.

Taking $k=j=2$, we have a function $\pi_{2}(X) \times \pi_{2}(X)$ $\rightarrow \pi_{3}(X)$, and this function is related to the coherence constraint for the composition operation $\circ_{0}: G_{2} \times_{0} G_{2} \rightarrow$ $G_{2}$. The axioms for a strict $n$-groupoid imply a uniqueness result for composition operations, while the Whitehead product can be interpreted in the categorical setting as the difference between two such operations. Uniqueness means that there is no difference, so the Whitehead products are all zero. Of course there are spaces with nonvanishing Whitehead products; the sphere $S^{2}$ is a primary example. Therefore, to define a fundamental $n$-groupoid $\Pi_{n} X$ that correctly models the homotopy $n$-type of an arbitrary space, we require a weak notion of $n$-groupoid instead of a strict one. One of the guiding principles for notions of weak $n$-groupoid is summed up in Grothendieck's homotopy hypothesis, formulated in his letter Pursuing Stacks.

Homotopy Hypothesis. The theory of weak $n$-groupoids is equivalent ${ }^{3}$ to that of homotopy $n$-types.

The homotopy hypothesis states a desired property of a proposed definition. It can be viewed through different lenses and has been the inspiration behind many lines of research in higher-dimensional category theory. On one end of the spectrum, the homotopy hypothesis can be the basis for a definition of weak $n$-groupoid, leading to something obviously tautological ("a weak $n$-groupoid is a homotopy $n$-type") but which yields little to no categorical

\footnotetext{
${ }^{3}$ In modern treatments, this is interpreted to mean an equivalence of homotopy theories, a notion we will not explain here.
} 
insight. At the other end of the spectrum, one can give a fully algebraic definition ${ }^{4}$ of weak $n$-categories (such as those in work of Batanin [Bat98] and Leinster [Lei04]) using the internal logic of category theory and then see if it satisfies the homotopy hypothesis.

The less like a topological space one defines a weak $n$ groupoid to be, the harder it is to verify the homotopy hypothesis and, as a consequence, the more interesting the methods involved are likely to be. There is currently no proof of the homotopy hypothesis for a fully algebraic definition of weak $n$-groupoid that is valid in all dimensions. There are proofs in the literature for some low-dimensional cases of fully algebraic definitions and systematic proofs valid across all dimensions for more topological definitions.

The theory of higher groupoids is situated within the larger theory of higher categories. As with weak $n$-groupoid, there is no single, all-purpose definition of weak $n$ category, and different authors have used a myriad of motivating principles for various applications. However, all current notions of weak $n$-category have the same sort of underlying data and composition laws as in Desideratum 9 but drop the requirement that all $i$-cells be invertible. We will return to this topic in the section "Stabilization in Category Theory" after establishing more of the relevant topology in the intervening sections.

\section{Stabilization in Topology}

For the remainder of this article we shift focus to stable phenomena in topology and category theory. In his book Infinite Loop Spaces, Adams writes, "A phenomenon is said to be stable if it occurs in any dimension, or any sufficiently large dimension, in a way which is essentially independent of the dimension." As we will see, the algebra of stable homotopy is closely connected with higher category theory. Topological suspension. In order to study phenomena that are independent of dimension, we need to understand the basic construction that shifts dimension.

Definition 11. Given a based space $(X, *)$, the suspension of $X$ is the based space $\Sigma X$ constructed by taking the cylinder $X \times I$ and identifying the subspaces $X \times\{0\}, X \times\{1\}$, and $\{*\} \times I$ to a single point, which then becomes the basepoint (see Figure 7).

Suspension increases the dimension of a space by 1 . In particular, one can check that there is a homeomorphism $\Sigma S^{n} \cong S^{n+1}$. Suspension is a functor from the category of based spaces to itself and is left adjoint to the loop space

\footnotetext{
${ }^{4} B y$ fully algebraic, we mean a definition that is equivalent to the category of algebras for a finitary monad on $(n-)$ globular sets. Defining the monad on globular sets as opposed to some other, more geometric, presheaf category ensures that all of the higher-categorical structure is encoded in the monad and not via some property of the objects in the underlying category/shapes involved.
}
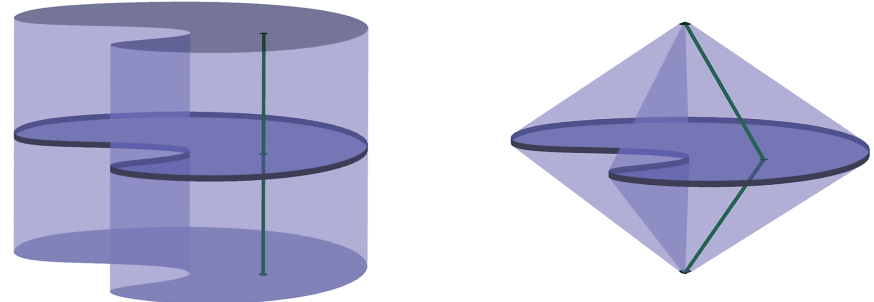

Figure 7. Cylinder and suspension; the subspace $\{*\} \times I$ is drawn as a line and is identified to a point in $\Sigma X$.

functor. That is, there is a natural correspondence between continuous maps $\Sigma X \rightarrow Y$ and continuous maps $X \rightarrow$ $\Omega Y$, giving an isomorphism known as the loop-suspension adjunction

$$
[\Sigma X, Y] \cong[X, \Omega Y] .
$$

Moreover, suspension is compatible with homotopies, so it induces a function $[X, Y] \rightarrow[\Sigma X, \Sigma Y]$.

A fundamental theorem in stable homotopy theory is that homotopy groups eventually stabilize, a corollary of the Freudenthal suspension theorem.

Theorem 12 (Freudenthal suspension theorem). Let $X$ and $Y$ be based $C W$-complexes and suppose $X$ has dimension $n \geq$ 1. Then the map

$$
\left[\Sigma^{k} X, \Sigma^{k} Y\right] \rightarrow\left[\Sigma^{k+1} X, \Sigma^{k+1} Y\right]
$$

is a bijection if $k \geq n+2$.

Letting $X=S^{n}$, one obtains an isomorphism

$$
\pi_{n+k}\left(\Sigma^{k} Y\right) \cong \pi_{n+k+1}\left(\Sigma^{k+1} Y\right)
$$

for $k \geq n+2$. Thus, the sequence

$$
\pi_{n}(Y) \rightarrow \pi_{n+1}(\Sigma Y) \rightarrow \pi_{n+2}\left(\Sigma^{2} Y\right) \rightarrow \cdots
$$

eventually stabilizes.

Definition 13. The stable term in the sequence above is the $n$th stable homotopy group of $Y$, denoted $\pi_{n}^{S}(Y)$.

Stable phenomena appear in cohomology, topological $K$-theory, and a host of other examples. In the 1950s, Spanier and Whitehead emphasized the study of stable topology by defining a stable homotopy category. The objects were defined to be finite CW complexes, and the set of maps from $X$ to $Y$, called the stable maps, was given by the direct limit

$$
\lim _{m \rightarrow \infty}\left[\Sigma^{m} X, \Sigma^{m} Y\right]
$$

Modern approaches to the stable homotopy category use one of the many equivalent notions of spectrum, which we now discuss. 
Topological spectra. A (topological) spectrum ${ }^{5} E$ consists of a sequence $\left\{E_{0}, E_{1}, \ldots\right\}$ of based spaces together with structure maps

$$
\sigma: \Sigma E_{n} \rightarrow E_{n+1} .
$$

For example, given a based space $X$, the suspension spectrum $\Sigma^{\infty} X$ is given by the sequence $\left\{X, \Sigma X, \Sigma^{2} X, \ldots\right\}$, with the structure maps given by identity maps. When $X=S^{0}$, we obtain the sphere spectrum, whose $n$th space is $S^{n}$.

A spectrum is an $\Omega$-spectrum if the structure maps' adjoints

$$
E_{n} \rightarrow \Omega E_{n+1}
$$

are homotopy equivalences for all $n$. We say that a space $X$ is an infinite loop space if it is the zeroth space of an $\Omega$ spectrum. Suspension spectra are generally not $\Omega$-spectra, but every spectrum is equivalent in a suitable sense to an $\Omega$-spectrum. The classical example of an $\Omega$-spectrum is the Eilenberg-Mac Lane spectrum $H A$ of an abelian group $A$. The $n$th space of $H A$ is given by the Eilenberg-Mac Lane space $K(A, n)$, and the adjoint structure maps are given by the homotopy equivalences $K(A, n) \simeq \Omega K(A, n+1)$ we discussed above.

We have noted previously that the $n$th cohomology group of a space $X$ with coefficients in the abelian group $A$ is isomorphic to the group of homotopy classes of maps from $X$ into $K(A, n)$ :

$$
H^{n}(X ; A) \cong[X, K(A, n)] .
$$

Using the loop-suspension adjunction (1), we recover the suspension isomorphism in cohomology: for $n>0$ we have

$$
\begin{aligned}
H^{n}(X ; A) & \cong[X, K(A, n)] \\
& \cong[X, \Omega K(A, n+1)] \\
& \cong[\Sigma X, K(A, n+1)] \cong H^{n+1}(\Sigma X ; A) .
\end{aligned}
$$

In fact, the most important features of cohomology with coefficients in $A$ can be recovered from the structure of the spaces $K(A, n)$, particularly as an $\Omega$-spectrum.

These properties characterize the groups $H^{*}(X ; A)$ and were originally written down by Eilenberg and Steenrod as a set of seven axioms, the first two of which constitute functoriality of $H^{*}(-; A)$. The final axiom is the dimension axiom, stating that the cohomology of a point is the coefficient group in dimension zero and vanishes elsewhere. Dropping the dimension axiom results in what are called generalized cohomology theories. The Brown representability theorem implies that all generalized cohomology theories are represented by $\Omega$-spectra just as $H^{*}(-; A)$ is represented by $H A$.

\footnotetext{
${ }^{5}$ The term "spectrum" in this sense is not motivated by any historical or mathematical relationship to its use in operator theory, ring theory, or algebraic geometry.
}

Spectra, as we have defined them, form a category $s p$, and $\Omega$-spectra form a full subcategory $\Omega s p$. While individual spectra are generally more complicated than individual spaces, the category $S p$ has many attractive features. In particular, there is a notion of homotopy between maps of spectra, leading to analogues of homotopy groups. Taking suspension spectra defines a functor from the category of based topological spaces to $S p$, and the stable maps between spaces are given precisely by maps in the category $S p$ between their suspension spectra. The stable homotopy groups of a based space are the homotopy groups in $S p$ of its suspension spectrum. Thus $S p$ generalizes the stable homotopy category constructed by Spanier and Whitehead.

We can reproduce the theory of Postnikov towers in this context, but now building the layers up using EilenbergMac Lane spectra and obtaining Postnikov invariants as stable maps. Stable Postnikov theory is simpler in some ways than its unstable predecessor: all groups are abelian, and the action of $\pi_{1}$ on higher homotopy is necessarily trivial. Yet it still contains a wealth of information, as we will see below.

\section{The Algebra of Iterated Loop Spaces}

Infinite loop space machines. One of the standard ways to construct cohomology theories with specific characteristics is to construct $\Omega$-spectra using one of the so-called "infinite loop space machines." A first step in constructing such an object is to determine when a space $E_{0}$ is homotopy equivalent to the space of loops on another space $E_{1}$.

Example 14. Let $G$ be a discrete group and let $B G$ be its classifying space. Then $G \simeq \Omega(B G)$. Thus, the classifying space is a construction of the Eilenberg-Mac Lane space $K(G, 1)$. One can construct further deloopings $B^{2} G=K(G, 2)$, etc., only under the further hypothesis that $G$ is abelian.

The equivalence $E_{0} \simeq \Omega E_{1}$ implies that $E_{0}$ has additional structure inherited from the algebra of loops. We can isolate the precise algebraic structure present on a loop space and use that to recognize when a given space has a delooping. For example, $\Omega E_{1}$ has a product induced by concatenation of loops. Although this product is not strictly associative, there is a canonical homotopy-for loops $a, b$, and $c$-between $(a b) c$ and $a(b c)$. This is the proof that multiplication in $\pi_{1}(X, x)$ is associative. These homotopies that witness associativity are themselves coherent; i.e., there is a family of homotopies mediating between the two composite homotopies and thus filling the pentagon in Figure 8 for any four loops $a, b, c, d \in \Omega E_{1}$.

In the case of a loop space, one can continue to fill with homotopies of higher dimensions, meaning that the product in $\Omega E_{1}$ is associative "up to all higher homotopies." Stasheff made this precise by constructing a family of 


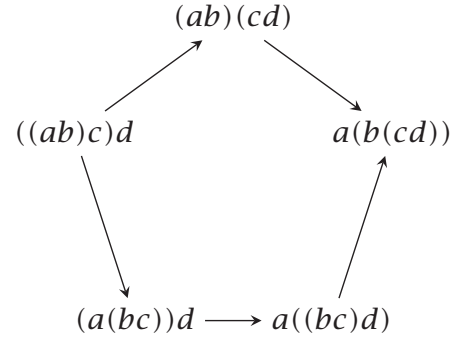

Figure 8. The associativity pentagon $K_{4}$.

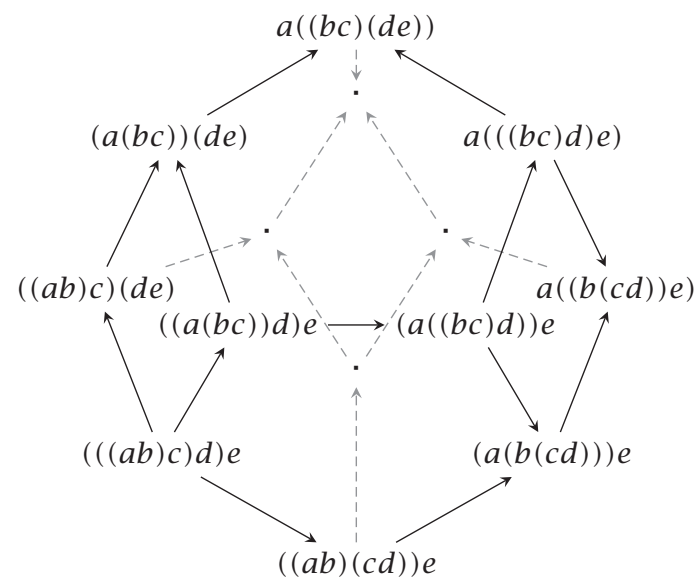

Figure 9. The associahedron $K_{5}$. The six pentagonal faces are instances of the pentagon $K_{4}$.

spaces $K_{n}$ that parametrize the homotopies that relate different parenthesizations of an $n$-fold product in the loop space. For each $n \geq 2, K_{n}$ can be realized as an $(n-2)$ dimensional polytope: $K_{2}$ is a point, $K_{3}$ is an interval, $K_{4}$ is the pentagon shown in Figure 8 , and $K_{5}$ is shown in Figure 9. These spaces are known as the associahedra and appear prominently in the combinatorics of polytopes.

Stasheff defined an $A_{\infty}$-space (meaning associative up to homotopies of all levels) to be a space $X$ together with maps $\mu_{n}: K_{n} \times X^{n} \rightarrow X$ that satisfy certain compatibility conditions with structure maps $K_{n} \times K_{m} \rightarrow K_{n+m-1}$. In particular, $X$ has a multiplication induced by $\mu_{2}: K_{2} \times$ $X^{2} \rightarrow X$, and the map $\mu_{3}: K_{3} \times X^{3} \rightarrow X$ encodes that it is homotopy associative, just as we have seen for concatenation in a loop space $\Omega E_{1}$. Stasheff proved that being an $A_{\infty}$-space is essentially all that is needed to construct a delooping.

Theorem 15. If $X$ is an $A_{\infty}$-space such that $\pi_{0}(X)$ is a group (with respect to the multiplication $\mu_{2}$ ), then there exists a based space $Y$ such that $X \simeq \Omega Y$.

A double loop space $\Omega^{2} Y$ has even more structure. Being a loop space, it has a multiplication that is associative up to all higher homotopies, and, moreover, this multiplication is commutative up to homotopy. The proof of this

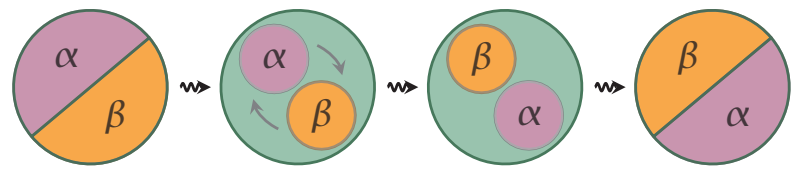

Figure 10. The Eckmann-Hilton argument; a family of reparametrizations of the disk.

fact is the same as the one used to prove that $\pi_{2}(Y, y)$ is abelian, known as the Eckmann-Hilton argument and sketched in Figure 10.

Extending the work of Stasheff on deloopings and $A_{\infty}$ spaces, one can ask whether there are necessary and sufficient conditions on a space that ensure it is homotopy equivalent to a double loop space or, more generally, an $n$-fold loop space for $n \geq 2$.

$E_{\infty}$ operads. May answered this question positively with his theory of operads in [May72].$^{6}$ An operad $P$ has spaces $P(k)$ encoding $k$-ary operations for each natural number $k$, together with composition maps combining operations of different arities. A $P$-algebra is a space $X$ together with maps

$$
P(k) \times X^{k} \rightarrow X
$$

that are compatible with the composition structure of $P$. This gives $X$ a collection of operations parametrized by $P$. May introduced the notion of $E_{n}$ operad for $1 \leq n \leq \infty$, with $E_{1}$ being equivalent to Stasheff's notion of $A_{\infty}$, and $E_{n}$ encoding $n$ commuting $E_{1}$ structures. He then proved that $E_{n}$ structures characterize $n$-fold loop spaces.

Theorem 16 (The recognition principle). If $X$ is an $E_{n}$ space such that $\pi_{0}(X)$ is a group, then there exists a based space $Y$ such that $X \simeq \Omega^{n} Y$.

If $X$ is an $E_{\infty}$ space such that $\pi_{0}(X)$ is a group, then, for each $n \geq 1$, there exists a based space $Y$ such that $X \simeq \Omega^{n} Y$.

The reader might be wondering about the condition that $\pi_{0}(X)$ is a group, since it has come up twice now. The space $X$ is said to be grouplike when this condition holds. The concatenation of loops is an operation on $\Omega Y$ under which every element has an inverse up to homotopy given by the loop going in reverse. Thus, $\Omega Y$ is grouplike for any space $Y$; this is the underlying reason that the fundamental group is a group, not merely a monoid. This seemingly pedestrian observation about loop spaces is more than a technicality. As we shall see below, invertibility in $\pi_{0}$ is essential to the categorical models of stable homotopy theory.

For $n \geq 2$, the recognition principle can be extended to more general $E_{n}$ spaces using the notion of topological group completion. Topological group completion universally extends $\pi_{0}$ to a group by adding inverses while localizing homology with respect to the action of $\pi_{0}$. It radi-

${ }^{6}$ Boardman and Vogt addressed the case $n=\infty$ using the theory of PROPs. 
cally changes the higher homotopy groups and is a progenitor of the stable homotopy groups of spheres and higher algebraic $K$-groups.

Categories with structure are an excellent way of producing $E_{n}$ spaces. Monoidal categories (i.e., categories with a functorial product that is associative and unital up to natural isomorphism) give rise to $E_{1}$ spaces upon taking classifying spaces, with braided and symmetric monoidal categories giving $E_{2}$ and $E_{\infty}$ spaces, respectively. ${ }^{7}$ The latter case follows from the work of May but was also independently proved by Segal using the theory of $\Gamma$-spaces. Thus, we have the following.

Slogan 17. To every symmetric monoidal category we can associate an $\Omega$-spectrum given by the group completion of its classifying space.

Example 18. Recall the category $\mathbf{S}$ introduced in Example 1 . This category is equivalent to the category of finite sets and isomorphisms and is symmetric monoidal using disjoint union. Its classifying space is then an $E_{\infty}$ space with $\pi_{0}(B \mathbf{S}) \cong \mathbb{N}$, and the connected component labeled by $n$ is an Eilenberg-Mac Lane space $K\left(S_{n}, 1\right)$.

The Barratt-Priddy-Quillen theorem proves that the spectrum associated to $\mathbf{S}$ is stably equivalent to the sphere spectrum. In particular, its homotopy groups are the stable homotopy groups of spheres.

Example 19. Let $R$ be a commutative ring and consider the category of finitely generated projective $R$-modules and module isomorphisms. This category is symmetric monoidal using direct sum. The associated spectrum is the $K$ theory spectrum, and its homotopy groups are the algebraic $K$-theory groups of $R$ defined by Quillen.

\section{Stabilization in Category Theory}

As discussed in the section "Higher Groupoids, More Subtle Algebra," one can motivate definitions of weak $n$-category by following the line of reasoning that led to Desideratum 9 for weak $n$-groupoids but omitting the invertibility requirement. We now explore another condition that every proposed definition should satisfy.

We have seen two perspectives on stable phenomena in topology: one via $E_{\infty}$ structures and the other via iterated loop structures, i.e., $\Omega$-spectra. The two are linked by the recognition principle, Theorem 16, which explains their relationship. The categorical reflection of these dual perspectives leads to another desired property for definitions of weak $n$-category, codified in the stabilization hypothesis below.

The stabilization hypothesis. In parallel with the two manifestations of topological stability, we identify two approaches to categorical stability. On the one hand, we have

\footnotetext{
${ }^{7}$ The reader may have expected $E_{3}$ instead of $E_{\infty}$ here, but being $E_{3}$ implies being $E_{\infty}$ due to the low categorical dimension.
}

a categorical analogue of an $E_{\infty}$ structure, given by the action of an appropriate operad in categories, or $n$-categories for $n \geq 1$. On the other hand, we have a categorical analogue of delooping by considering $(n+k)$-categories with trivial cells in dimensions 0 through $k-1$; this means a single object, a single 1-cell that is necessarily the identity, a single 2-cell that is the identity on the identity, and so on. These are known as $k$-degenerate $(n+k)$-categories, and they carry additional algebraic structure as a consequence of the low-dimensional degeneracy.

For a $k$-degenerate $(n+k)$-category, the composition laws $\circ_{0}, \circ_{1}, \ldots, \circ_{k-1}$ produce $k$ different, yet intertwined, multiplications on the $n$-category obtained by treating the $k$-cells as the new objects, the $(k+1)$-cells as the new 1 cells, and so on. This is an algebraic analogue of the multiplicative structure one obtains on a $k$-fold loop space, and one major research focus is the extent to which one can describe this structure algebraically. For example, in the case $k=1, n=0$, we have a 1 -category with a single 0 cell and a set of 1-cells. Composition of morphisms equips this set with the structure of a monoid. In the case $k=$ $2, n=0$, we have a 2-category with a single 0 - and 1-cell, and the 2-category structure equips the set of 2-cells with the structure of a commutative monoid. The argument proving commutativity is once again the Eckmann-Hilton argument (Figure 10).

A study of higher categories by Baez and Dolan suggests that $(k+1)$-degenerate $(n+k+1)$-categories should have a more commutative structure than $k$-degenerate $(n+k)$ categories and further that this phenomenon should eventually yield a stable notion of symmetric monoidal higher category, corresponding to the stability indicated by the Freudenthal suspension theorem.

Stabilization Hypothesis. The theory of $k$-degenerate $(n+$ $k)$-categories is equivalent ${ }^{8}$ to that of $(k+1)$-degenerate $(n+$ $k+1$ )-categories for $k \geq n+2$.

As with the homotopy hypothesis, the stabilization hypothesis is a desired property of a proposed definition of $n$-category. One can explore its validity for a variety of notions of higher category, and as before the general case for fully algebraic notions of higher categories is unsettled [CG07, CG11]. One can also approach this question using more topological notions of higher category and describe their properties in homotopical terms. For these homotopical analogues of the stabilization hypothesis see, e.g., Lurie [Lur09], Batanin [Bat17], Simpson [Sim98], GepnerHaugseng [GH15].

The notions of stable $n$-category (via the stabilization hypothesis) and $E_{\infty} n$-category are related by an intermediate notion not appearing distinctly among topological

\footnotetext{
${ }^{8}$ As with the homotopy hypothesis, the notion of equivalence here is subject to interpretation and beyond the scope of this article.
} 


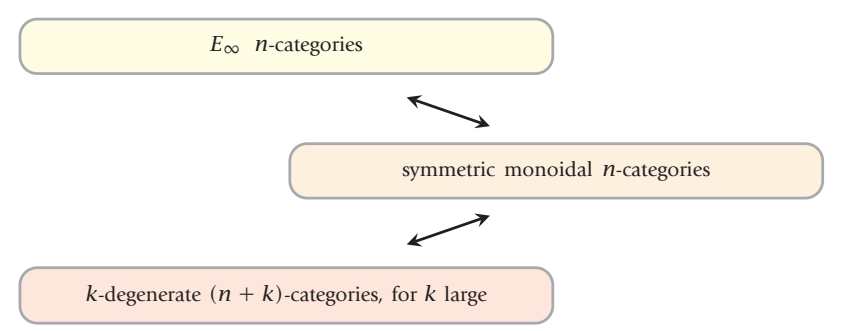

Figure 11. Coherence theorems for symmetric monoidal $(n$-)categories serve to unify the two notions of stabilization in categorical algebra.

spaces. This is the notion of symmetric monoidal $n$-category, an $n$-category equipped with a monoidal structure which is associative, unital, and commutative up to invertible 1cells which are coherent up to invertible 2-cells which are coherent up to ... until dimension $n$. It is this notion that arises most directly in applications and should be considered the primary algebraic concept. This is depicted in Figure 11 .

In the previous section we mentioned the case $n=$ 1; Mac Lane's coherence theorem implies that categories equipped with $E_{\infty}$ structures are the same, up to equivalence, as symmetric monoidal categories. However, it is not at all clear how the relationship between $E_{\infty}$ and symmetric monoidal generalizes to higher categories. Higherdimensional coherence theorems are needed to relate these to each other and to $k$-degenerate $(n+k)$-categories. The stable homotopy hypothesis. Having explored some categorical analogues of stable phenomena, we seek a conceptual explanation for these, a stable analogue of the homotopy hypothesis. To explore this, we must first determine the correct structures to compare. The stable notion of homotopy $n$-type is that of stable homotopy $n$-type: a spectrum whose stable homotopy groups vanish in negative degrees and those greater than $n$. The stable notion of weak $n$-groupoid is known as a Picard $n$-category and has three key properties:

- it has an underlying weak $n$-groupoid;

- it is a symmetric monoidal $n$-category;

- it is grouplike, meaning that every object is invertible with respect to the monoidal product.

The first of these corresponds, topologically, to an underlying unstable $n$-type. The second corresponds to an $E_{\infty}$ structure, and the third corresponds to the fact that the zeroth space of any spectrum is grouplike. Thus we have the following formulation.

Stable Homotopy Hypothesis. The theory of Picard $n$-categories is equivalent to that of stable homotopy n-types.

\section{Low-Dimensional Examples of the Stable Homo-} topy Hypothesis

We will examine some low-dimensional cases of the stable homotopy hypothesis. When $n=0$, the topological side consists of stable homotopy 0-types-the EilenbergMac Lane spectra $H A$. On the categorical side we have Picard 0 -categories, which are weak 0 -groupoids (such a thing is just a set) together with an $E_{\infty}$ structure (giving a commutative monoid) such that every object is invertible (thus, an abelian group). In other words, stable homotopy 0 -types are classified, up to stable equivalence, by a single abelian group, $\pi_{0}$, and Picard 0 -categories are abelian groups. The stable homotopy hypothesis in dimension zero follows as an exercise in writing out the various definitions carefully.

The 1-dimensional stable homotopy hypothesis. The case $n=1$ is more interesting and goes back to Sính [Sín78]. On the topological side, a stable homotopy 1 type is a spectrum $X$ with all homotopy groups vanishing except $\pi_{0} X$ and $\pi_{1} X$. These abelian groups alone do not determine the stable 1-type. We also need a single Postnikov invariant $k_{0}$, which is a priori given by a homotopy class of maps of spectra $H\left(\pi_{0} X\right) \rightarrow \Sigma^{2} H\left(\pi_{1} X\right)$. Via a group cohomology calculation, one can show that the set of such homotopy classes is naturally isomorphic to the set of group homomorphisms $\pi_{0} X \otimes \mathbb{Z} / 2 \rightarrow \pi_{1} X$. On the categorical side, a Picard 1-category is a weak 1-groupoid (which just means an ordinary groupoid) together with an $E_{\infty}$ structure (which makes it into a symmetric monoidal category) such that every object is invertible (so for any $x$, there exists $y$ such that $x \otimes y \cong 1$ and $y \otimes x \cong 1$ ).

The key to comparing these two structures is the following observation. A Picard 1-category $P$ has naturally arising homotopy groups with $A_{0}=\pi_{0} P$ being the set of isomorphism classes of objects and $A_{1}=\pi_{1} P$ being the set of automorphisms of the unit object. We can also construct a homomorphism $h_{0}: A_{0} \otimes \mathbb{Z} / 2 \rightarrow A_{1}$ from the symmetry of $P$ : given an object $x, h_{0}(x)$ is an automorphism of the unit determined by the symmetry isomorphism $x \otimes x^{-1} \cong x^{-1} \otimes x$. These categorical invariantsobjects, automorphisms, and $h_{0}$-correspond to topological invariants-two homotopy groups and the Postnikov invariant $k_{0}$ discussed in the section "Higher Homotopy Groups, More Subtle Invariants." Work of the second and third authors [JO12] discusses this more thoroughly and provides a proof of the following result.

Theorem 20 ([JO12]). Let $P$ be a Picard 1-category. The classifying space BP is a stable 1-type. The stable homotopy groups and single Postnikov invariant of $B P$ are those computed from $P$ above.

The correspondence between topological and categorical data is summarized in Figure 12. With a little addi- 


\begin{tabular}{l|l|l}
$n$ & Stable $n$-types & Picard $n$-categories \\
\hline 0 & Abelian group $\pi_{0}$ & Abelian group of objects, $A_{0}$ \\
1 & $\pi_{0}, \pi_{1}$, and & $A_{0}, A_{1}$ \\
& $k_{0}: \pi_{0} \otimes \mathbb{Z} / 2 \rightarrow \pi_{1}$ & $h_{0}: A_{0} \otimes \mathbb{Z} / 2 \rightarrow A_{1}$ \\
& & \\
& $\pi_{0}, \pi_{1}, \pi_{2}$ & $A_{0}, A_{1}, A_{2}$ \\
$k_{0}: \pi_{0} \otimes \mathbb{Z} / 2 \rightarrow \pi_{1}$ & $h_{0}: A_{0} \otimes \mathbb{Z} / 2 \rightarrow A_{1}$ \\
$k_{1} i_{1}: \pi_{1} \otimes \mathbb{Z} / 2 \rightarrow \pi_{2}$ & $h_{1}: A_{1} \otimes \mathbb{Z} / 2 \rightarrow A_{2}$ \\
$k_{1}$ & currently unknown!
\end{tabular}

Figure 12. Low-dimensional data for stable homotopy types and higher Picard categories.

tional work, we have the following.

Corollary 21. The 1-dimensional stable homotopy hypothesis holds for Picard categories as defined above.

We mention two features of the case $n=1$ before moving on to $n=2$. The first is that it is useful to consider Picard 1-categories that are strict and skeletal, meaning that their product is strictly associative and unital, and every isomorphism is an automorphism; i.e., there are no isomorphisms between distinct objects. We thus picture a strict and skeletal Picard 1-category as a discrete abelian group of objects with an abelian group of automorphisms over each object.

Example 22. Consider a skeletal Picard category whose objects are the group $\mathbb{Z} / 6$ and each object has automorphism group $\mathbb{Z} / 2$. There are two such Picard categories: one with trivial symmetry and the other with symmetry $x+y \cong$ $y+x$ given by $\sigma^{x y}$. These correspond to the two homomorphisms $\mathbb{Z} / 6 \otimes \mathbb{Z} / 2 \rightarrow \mathbb{Z} / 2$.

These abelian groups of automorphisms are necessarily all isomorphic as a consequence of every object being invertible. One can prove that every Picard 1-category is equivalent to a strict skeletal one, but it is worth noting that dropping the symmetry from the definition of a Picard 1-category will cause the proof of this strict skeletal approximation to fail. In fact, the nonsymmetric version has a different algebraic classification in which the associativity isomorphisms correspond to 3-cocycles for group cohomology (see Baez-Lauda [BL04], Joyal-Street [JS93]).

The second feature to mention is that such a fully algebraic classification allows one to construct specific spectra in new ways. The sphere spectrum is a central object of study in algebraic topology, and it is easy to compute that its stable 1 -type is determined by $\pi_{0} \cong \mathbb{Z}, \pi_{1} \cong \mathbb{Z} / 2$, and the unique isomorphism $k_{0}: \mathbb{Z} \otimes \mathbb{Z} / 2 \cong \mathbb{Z} / 2$. This technique gives a concrete and fully algebraic construction of the 1-type of the sphere spectrum. These Postnikov data appear in applications to quantum field theories as the Picard 1-category of invertible graded abelian groups and isomorphisms; see, e.g., Kapranov [Kap15] or Freed [Fre14]. One can also show ([JO12]) that this is the free Picard 1-category generated by a single object, giving the 1-truncated sphere spectrum an interesting universal property.

Together, these two features and their analogues for $n>$ 1 are examples of the following.

Slogan 23. Definitions of Picard n-category for which the stable homotopy hypothesis holds will explicitly encode the algebraic invariants that characterize the stable $n$-type of a space or spectrum.

The 2-dimensional stable homotopy hypothesis. A proof of the stable homotopy hypothesis in dimension 2 appears in recent work of the authors [GJO19]. This uses a notion of Picard 2-category defined in [GJO17, GJOS17] that is fully algebraic and yet general enough to realize all stable 2-types.

Theorem 24 ([GJO19]). There exists a fully algebraic notion of Picard 2-category for which the 2-dimensional stable homotopy hypothesis holds.

One can now ask about the Postnikov theory of Picard 2-categories. Although we will not give the definition of Picard 2-category here, we can describe much of the characterizing data and its role in an associated Postnikov tower. First, a Picard 2-category $P$ is a grouplike symmetric monoidal 2-groupoid. This implies we have three abelian groups known as the algebraic homotopy groups of $P$ :

- $A_{0}$ is the set of equivalence classes of objects in $P$.

- $A_{1}$ is the set of isomorphism classes of 1-cell endomorphisms of the unit object in $P$.

- $A_{2}$ is the set of 2-cell endomorphisms of the identity 1-cell on the unit object of $P$.

We can also identify two Picard 1-categories associated with $P$. First, there is the truncation $P_{1}$ consisting of the objects of $P$ and the isomorphism classes of the 1-cells of $P$. The symmetric monoidal structure on $P$ makes $P_{1}$ a Picard 1-category. Second, there is the category of 1- and 2-cells over the unit in $P$. This is the category of functors and natural transformations from the free loop category into $P$ and is denoted $\Omega P$.

The symmetric monoidal structure on $P$ also makes $\Omega P$ a Picard 1-category. Then by the theory of Picard 1-categories there are two algebraic Postnikov invariants, which we denote by $h_{0}$ and $h_{1}$. From $P_{1}$ we have $h_{0}: A_{0} \otimes$ $\mathbb{Z} / 2 \rightarrow A_{1}$, and from $\Omega P$ we have $h_{1}: A_{1} \otimes \mathbb{Z} / 2 \rightarrow A_{2}$. These are also shown in Figure 12. The following result summarizes what we currently know about the Postnikov data of Picard 2-categories. 


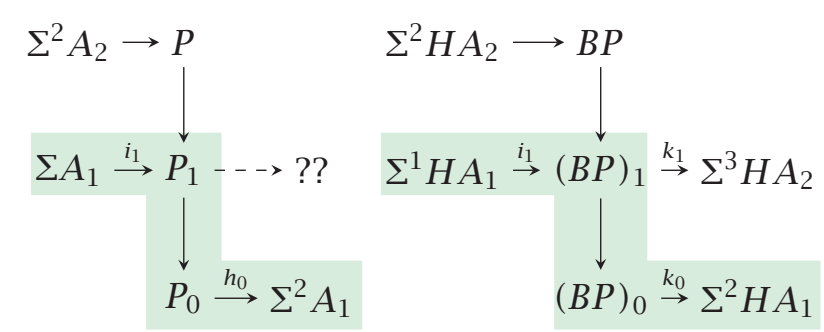

Figure 13. Stable Postnikov towers for $P$ and $B P$.

Theorem 25 ([GJO17, GJOS17]). Let P be a Picard 2-category, with classifying space $B P$.

- The symmetric monoidal structure on $P$ gives $B P$ an infinite loop space structure.

- The spectrum associated to BP is a stable 2-type.

- The homotopy groups of BP are given by the algebraic homotopy groups of $P$.

- The bottom Postnikov invariant of $B P, k_{0}$, is given by $h_{0}$, the symmetry of $P_{1}$.

- The composite $k_{1} i_{1}$ is given by $h_{1}$, the symmetry of $\Omega P$ (see Figure 13).

Given an abelian group $A$, we implicitly regard this as a Picard 1- or 2-category with objects given by $A$ and trivial higher morphisms. We let $\Sigma A$ denote the Picard 1category with a single object and with endomorphism group $A$. This is a 1-degenerate $(0+1)$-groupoid, and its classifying space is a $K(A, 1)$. We let $\Sigma^{2} A$ denote the Picard 2-category with a single object, single 1-cell, and endomorphism 2-cells given by $A$. This is a 2-degenerate $(0+2)$ groupoid, and its classifying space is a $K(A, 2)$. With this notation, the content of Theorem 25 is expressed in a partial Postnikov tower for $P$ in Figure 13.

The missing pieces in Figure 13, which one would label $k_{1}$ and $\Sigma^{3} A_{2}$, require a theory of symmetric monoidal 3-categories: following the stabilization hypothesis, the natural categorical construction of $\Sigma^{3} A_{2}$ should be as a symmetric monoidal 3-category with a single object, 1-cell, and 2-cell, and 3-cells given by the elements of $A_{2}$. This is the subject of future work. The fifth bullet point of Theorem 25 is a partial result in this direction, but does not give complete information about $k_{1}$.

Most of the steps outlined in the 1-dimensional case have a 2-dimensional analogue. Although the proofs of these results require more sophisticated categorical techniques, the central technical tool is the coherence theory of symmetric monoidal 2-categories as begun by the first and third authors in [GO13], continued in [SP11], and further refined in [GJO17].

Slogan 26. The identification of homotopical structure with algebraic structure is an application of categorical coherence theory.

\section{Open Problems}

A key feature of the 1-dimensional stable homotopy hypothesis is the correspondence between the single Postnikov invariant $k_{0}$ and the symmetry. However, we do not have a 2-dimensional analogue that fully explains the Postnikov invariant $k_{1}$; this motivates a series of related problems.

Problem 27. Extend the work above to a complete interpretation of $k_{1}$ in terms of the categorical structure in a Picard 2-category. Use this to explore some interesting examples from topology.

Problem 28. Develop a theory of kernels and cokernels for Picard $n$-categories and relate it to fiber/cofiber sequences in stable homotopy.

Problem 29. Identify a Picard 2-category that models the 2-type of the sphere, along the lines of Kapranov's algebraic model. This requires a connection between algebraic sign conventions and the Postnikov invariants of the sphere spectrum.

Problem 30. Develop a homological algebra for Picard 1categories and relate it to Postnikov obstructions.

Problem 31. Use the algebra of Picard 1- and 2-categories to study homotopy operations.

\section{References}

[BL04] Baez JC and Lauda AD, Higher-dimensional algebra. V. 2-groups, Theory Appl. Categ. 12 (2004), 423-491. MR2068521

[Bat98] Batanin MA, Monoidal globular categories as a natural environment for the theory of weak $n$-categories, $A d v$. Math. 136 (1998), no. 1, 39-103. MR1623672

[Bat17] Batanin MA, An operadic proof of Baez-Dolan stabilization hypothesis, Proc. Amer. Math. Soc. 145 (2017), no. 7, 2785-2798. MR3637930

[CG07] Cheng E and Gurski N, The periodic table of $n$ categories for low dimensions. I. Degenerate categories and degenerate bicategories, Categories in algebra, geometry and mathematical physics, Contemp. Math., vol. 431, Amer. Math. Soc., Providence, RI, 2007, pp. 143-164. MR2342826

[CG11] Cheng E and Gurski N, The periodic table of $n$ categories II: Degenerate tricategories, Cah. Topol. Géom. Différ. Catég. 52 (2011), no. 2, 82-125. MR2839900

[Fre14] Freed DS, Anomalies and invertible field theories, String-Math 2013, Proc. Sympos. Pure Math., vol. 88, Amer. Math. Soc., Providence, RI, 2014, pp. 25-45. MR3330283

[GH15] Gepner D and Haugseng R, Enriched $\infty$-categories via non-symmetric $\infty$-operads, Adv. Math. 279 (2015), 575-716. MR3345192

[GJO17] Gurski N, Johnson N, and Osorno AM, K-theory for 2-categories, Advances in Mathematics 322 (2017), 378-472. MR3720802 


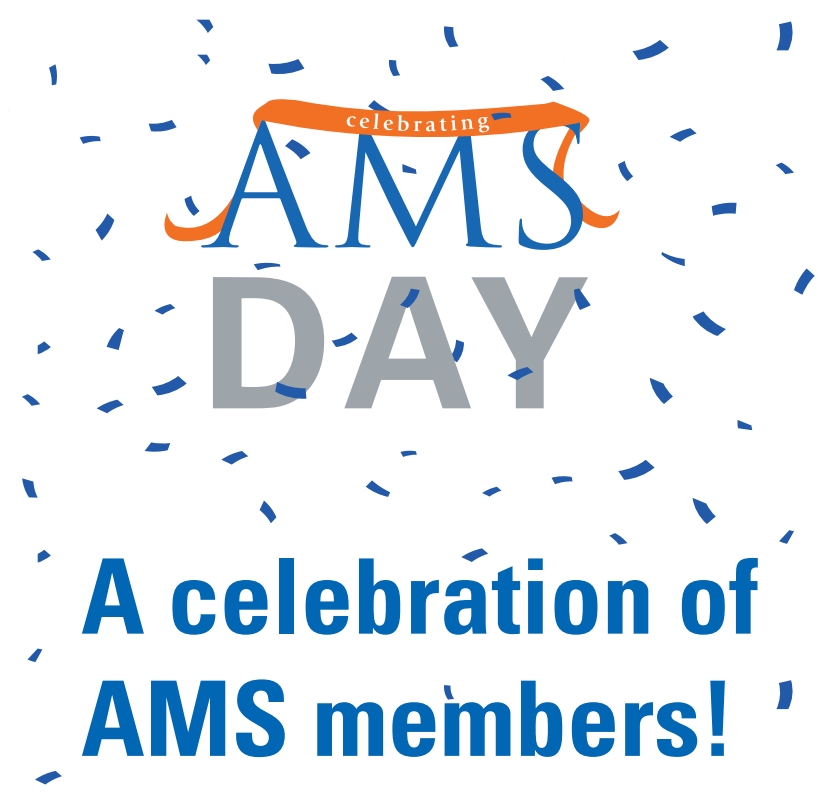

Join us on Wednesday, October 16,

- 2019 as we honor our AMS members

via "AMS Day", a day of specials on

AMS publications, membership, and

- more! Stay tuned on Facebook, Twitter,

\section{and member emails for details about}

this exciting day. Spread the word

- about \#AMSDay today!
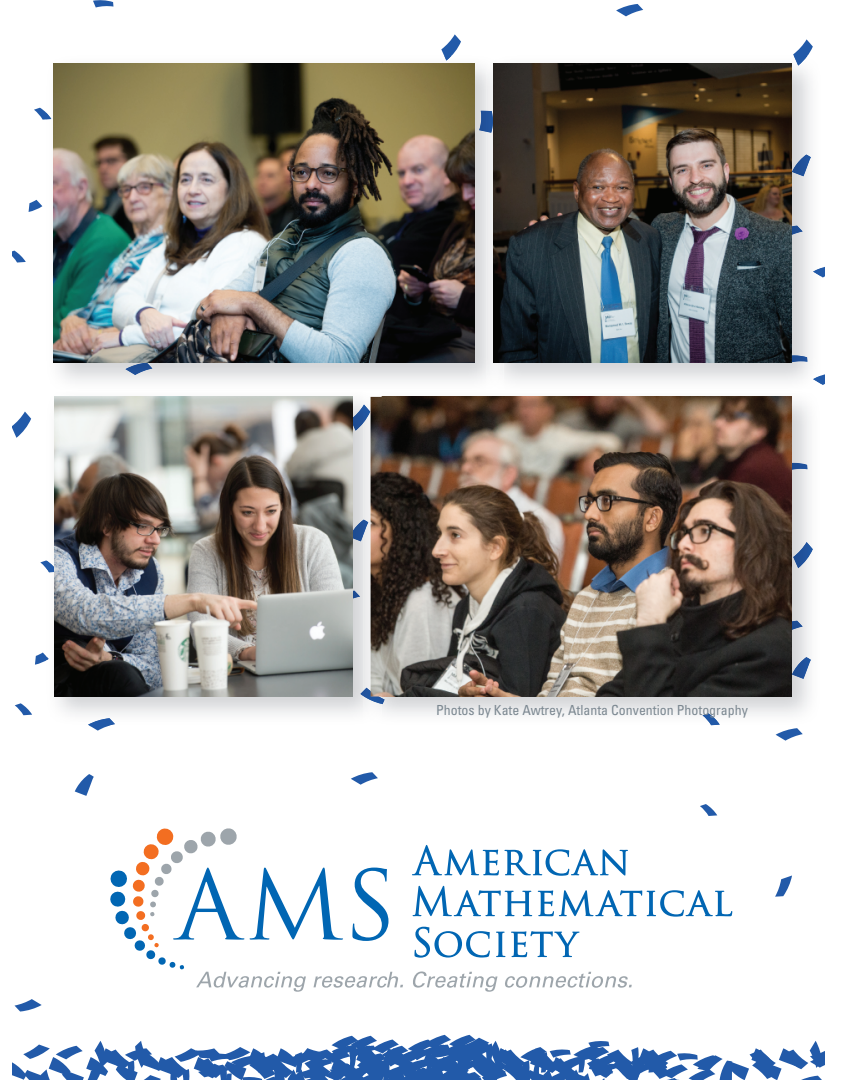

[GJO19] Gurski N, Johnson N, and Osorno AM, The 2dimensional stable homotopy hypothesis, J. Pure Appl. Algebra 223 (2019), no. 10, 4348-4383. MR3958095

[GJOS17] Gurski N, Johnson N, Osorno AM, and Stephan M, Stable Postnikov data of Picard 2-categories, Algebr. Geom. Topol. 17 (2017), 2763-2806. MR3704242

[GO13] Gurski N and Osorno AM, Infinite loop spaces, and coherence for symmetric monoidal bicategories, Adv. Math. 246 (2013), 1-32. MR3091798

[JO12] Johnson N and Osorno AM, Modeling stable onetypes, Theory Appl. Categ. 26 (2012), No. 20, 520-537. MR2981952

[JS93] Joyal A and Street R, Braided tensor categories, Adv. Math. 102 (1993), no. 1, 20-78. MR1250465

[Kap15] Kapranov M, Supergeometry in mathematics and physics, arXiv:1512.07042v1, 2015.

[Lei04] Leinster T, Higher operads, higher categories, London Mathematical Society Lecture Note Series, vol. 298, Cambridge University Press, Cambridge, 2004. MR2094071

[Lur09] Lurie J, Higher topos theory, Annals of Mathematics Studies, vol. 170, Princeton University Press, Princeton, NJ, 2009. MR2522659

[May72] May JP, The geometry of iterated loop spaces, Lectures Notes in Mathematics, Vol. 271, Springer-Verlag, BerlinNew York, 1972, MR0420610

[SP11] Schommer-Pries C, The classification of twodimensional extended topological field theories, arXiv:1112.1000v2, 2011.

[Sim98] Simpson C, On the Breen-Baez-Dolan stabilization hypothesis for Tamsamani's weak $n$-categories, arXiv:math/9810058, 1998.

[Sín78] Sính HX, Gr-catégories strictes, Acta Mathematica Vietnamica (1978), 47-59. MR527303

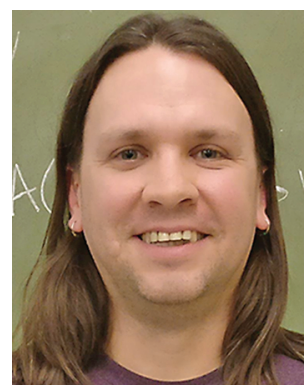

Nick Gurski

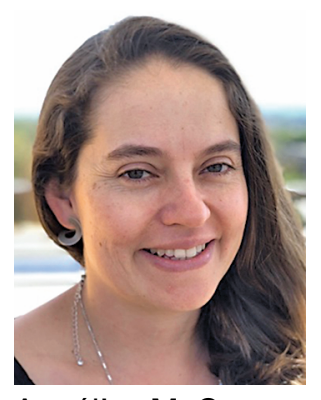

Angélica M. Osorno

Credits

All figures and author photos are courtesy of the authors. 